\title{
Spectrophotometric Studies of Ternary Complexes of Erbium to Explore the Hyperchromic Effect with Various Ligands and their use in Microdetermination
}

\author{
SHAMIM SHAUKAT KHAN \\ Department of General Education, Dar Al-Hekma University, Jeddah, Kingdom of Saudi Arabia. \\ ${ }^{*}$ Corresponding author E-mail: skhan@ dah.edu.sa \\ http://dx.doi.org/10.13005/ojc/360616
}

(Received: September 11, 2020; Accepted: November 13, 2020)

\begin{abstract}
A comprehensive spectrophotometric study is undertaken for ternary complexes of higher rare earth element Erbium with ethylene diamine tetra acetic acid (EDTA), hydroxyl ethylene diamine triacetic acid (HEDTA), trans 1, 2, cyclohexane diamine tetra acetic acid (CYDTA) and ethylene glycol bis (2-amino ethylene) $\mathrm{N}, \mathrm{N}^{1}, \mathrm{~N}^{11}, \mathrm{~N}^{111}$ tetra acetic acid (EGTA) as primary ligand and the binary ligands used are Maltol, Tiron, Kojic acid and $\alpha$-Picolinic acid. The ternary complexes shows hyperchromic effect as compare to primary complexes which is exploited to determine the stoichiometry of ternary complexes and it is found to be 1:1:1. The conditional stability constants $\left(\mathrm{K}_{\mathrm{s}}{ }^{1}\right)$ are corrected using $\mathrm{k}$ $H$ values of ligands. The order of stability with the series of ligands in different systems is deduced as EDTA and HEDTA: $\alpha$ Picolinic acid $>$ Tiron $>$ Kojic acid $>$ Maltol and CYDTA and EGTA: $\alpha$ Picolinic acid > Kojic acid > Tiron > Maltol. The \% increase in molar coefficients of mixed ligand complexes of Erbium is significant which can facilitates the analytical measurements for its micro determination.
\end{abstract}

Keywords: Ternary complexes, Molar extinction coefficients, Primary complex, Erbium, Stability constant, Molar ratio and absorbance.

\section{INTRODUCTION}

In past years there have seen a rise in the study of mixed ligand or ternary complexes, where a metal ion is held simultaneously by two different ligands. However, there is a large paucity of data of such complexes with rare earth metals. This is because the study of rare earth complexes encounters several difficulties. In these elements, the electrons enter into the deeper $f$ orbitals inside the atom and they have small magnitude for $\mathrm{f}$-f transition. Therefore, the molar extinction coefficients of rare earth complexes are smaller compare to transition metals. Further, their spectral characteristics are not much influenced by the chemical environment as they have single stable oxidation state of +3 . Thus, attention is directed towards investigating ternary systems (mixed ligand complexes) of rare earth elements with spectrophotometric technique. The rare earth metal ions from solution are separated, estimated and analyzed using versatile complexing agents to form mixed ligand or ternary complexes with constant and extended coordination. Such ternary complexes of $\mathrm{Ho}^{+3}$ and $\mathrm{Er}^{+3}$ mixed chelates

This is an Open Access article licensed under a Creative Commons license: Attribution 4.0 International (CC- BY). Published by Oriental Scientific Publishing Company @ 2018

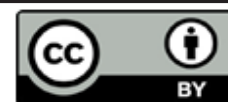


with various complexones and 5-sulphosalicylic acid have been studied by Taketatsu and Yoshida ${ }^{1}$ at higher $\mathrm{pH}$ of 10.7 to 11.0 whereas the present study is at moderate $\mathrm{pH}$ 8.0.

Potentiometric studies of mixed ligand complexes of $\mathrm{La}^{+3}$ with EDTA and 2, 3 dihydroxy benzoic acids and their stability constants are determined by Cam, Irez and Aydin. ${ }^{2}$

Sequential determination of thorium and rare earths elements with EDTA and Kojic acid are more stable than xylenol orange ternary complexes ${ }^{3}$.

Potentiometric studies of mixed ligand complexes of rare earth reported by Khan and Reddy4 with EDTA as primary and TPP and ATP as secondary ligands.

Spectrophotometric studies of coordination ternary complexes of rare earth elements with chrome azurol in presence of Cetylpyridinium bromide with $\lambda_{\max } 620 \mathrm{~nm}$ at $\mathrm{pH} 5.4$ for the determination of Lanthanum, Praseodymium, Neodymium, Samarium, Gadolinium and Dysprosium is reported by Vekhande and Munshi ${ }^{5}$. Sensitization of Eriochromeazurol-B in presence of Cetyldimethylethylammonium Bromide for the Microdetermination of Some Lanthanides is reported by Zade, kalbende, Umerkar and Belsare. ${ }^{6}$

In this paper, spectrophotometric studies of ternary mixed ligand complexes of higher rare earth element (Erbium) are undertaken with hexadentate primary ligands. The primary ligands used are: EDTA, ethylene diamine tetra acetic acid, HEDTA, hydroxyl ethylene diamine triacetic acid, CYDTA, trans-1,2-cyclohexane diamine tetra acetic acid and EGTA ethylene glycol bis (2, amino ethylene)N, $\mathrm{N}^{1}, \mathrm{~N}^{11}, \mathrm{~N}^{111}$ tetra acetic acid. Further, the secondary ligands, which are bidentate, are Maltol, Tiron, Kojic acid and $\alpha$-Picolinic acid. In the present study the hyperchromic effect that is increased in absorption intensities exhibited by the rare earth elements in mixed ligand complexes as compared with the primary complexes are exploited. This is evident from the increased in molar extinction coefficient values of mixed ligand or ternary complexes $(\mathrm{Em})$ as compared with the molar extinction coefficients of binary complexes (Ep). The stability constants and the stoichiometry of ternary complexes are also determined for the systems under study.
In utilizing the rare earth absorption bands for analytical purposes, the first problem is to choose the most suitable band for use as index peak to calculate the extinction coefficients under specified test conditions. The light absorption bands of rare earth elements are extremely narrow and consequently, a number of readings should be taken around each index peak position to ensure that the point of maximum light absorption is seen. A generalized light absorption background is present especially in shorter wavelength, so the readings taken may be incorrect if the analyst is unaware of the background due to impurities. Thus, an automatic recording spectrophotometer is used for rare earth analysis.

\section{Methodology}

In the present study, the modified molar ratio method $^{7}$ is used which gives the curve that rises as a straight line and breaks sharply to almost constant optical density at the molar ratio of components in the complex. The primary complex of erbium and hexadentate ligand is a primary species. Equimolar mixture of erbium ions and primary ligand solution is mixed with various concentrations of the secondary ligand. The spectra are studied between fixed wavelength ranges on Hitachi 330 automatic recording spectrophotometer. The optical densities or absorptions are measured at $\lambda_{\max }$ values and the optical density is plotted against the mole ratio concentration of secondary ligand (LS) to the concentration of primary complex (Ln-Y).

$$
[L s] /[L n-Y]
$$

Where Ls is secondary ligand and Ln-Y as primary complex. The extinction coefficient for primary complex $\left(E_{p}\right)$ and ternary complex $\left(E_{m}\right)$ are calculated using Lambert-Beer's law.

$A=E_{p} \times C \times L$

Where $E_{p}$ is the molar extinction coefficient of primary complex, $\mathrm{C}$ is the molar concentration, $\mathrm{L}$ is the optical path length in $\mathrm{cm}$ and $\mathrm{E}_{\mathrm{m}}$ is the molar extinction coefficient for ternary complex. For ternary complex extinction coefficient $\left(\mathrm{E}_{m}\right)$ is computed at the sharp break point in the curve of absorbance versus mole ratio. 
Further, the concentration of the ternary complexes and their stability constants were computed from the absorbance values on the rising portion of the curve of absorbance versus the mole ratio of [Ls]/[primary complex] relation given by Shetty and Sathe. ${ }^{8}$

$A=E_{p}(p-x)+E_{m}{ }^{*} x$

Where ' $A$ ' is the measured absorbance of mixed or ternary complex at the respective $\lambda_{\text {max }}$ values. $E_{p}$ is the extinction coefficient of primary complex, which is a mixture of rare earth element and polyamino carboxylic acids in equimolar quantity; $E_{m}$ is the molar extinction coefficient of metal ion-polyamino carboxylic acids and bidentate secondary ligand. ' $p$ ' is the initial concentration of primary ligand and ' $x$ ' is the concentration of mixed chelate formed.

The conditional stability constants at the $\mathrm{pH}$ of measurement calculated by the following equation:

$\mathrm{K}_{\mathrm{s}}{ }^{1}=\mathrm{x} /(\mathrm{p}-\mathrm{x})(\mathrm{b}-\mathrm{x})$

Where $b$ is the concentration of secondary ligand added, and $\mathrm{K}_{\mathrm{s}}{ }^{1}$ is the stability constant at $\mathrm{pH}$ value of $8 \pm 0.05$.

\section{Apparatus used}

The details of the apparatus utilized for analysis mentioned below.

\section{Spectrophotometer}

Make: Hitachi model 330 automatic recording spectrophotometer.

Measuring wavelength range: $187-2500 \mathrm{~nm}$

Wavelength display: 4 digits LED

Wavelength accuracy: $\pm 0.2 \mathrm{~nm}$

Photometric accuracy: 0.002 ABS

Recording mode: overlapped or sequential

Light source: WI or $\mathrm{D}_{2}$ lamp automatic

Other details: The instrument performs the checks of performance and functions as well as calibrating operations. The instrument standardized by using distilled water as blank and potassium permanganate. The spectra run at a scan speed of $60 \mathrm{~nm} /$ minute. The response adjusted at one and the slit width $2 \mathrm{~nm}$. The scanning wavelength range for erbium $450-350 \mathrm{~nm}$ and on the spectra the absorbance at $\lambda_{\max }$ chosen for the maximum sensitivity for all calculations.

\section{$\mathrm{pH}$ meter} $0.02 \mathrm{pH}$ unit.

Make: M64 Research pH -meter, accuracy:

\section{Preparation of solutions}

The method of standardization of metal ions solutions and the primary ligands are accepted methods from Vogel, A.I. ${ }^{9}$. Further secondary ligands are crystallised from appropriate solvents.

\section{Standard zinc Solution}

Standard zinc solution $0.1 \mathrm{M}$ is prepared by dissolving $1.6343 \mathrm{~g}$ of $\mathrm{BDG}$ Analar R zinc pellets in hydrochloric acid and volume made to $250 \mathrm{~mL}$.

EDTA (Ethylene diamine tetra acetic acid)

Disodium dihydrate E GR, made by Merck, dried at $80^{\circ} \mathrm{C}$ to prepare a $0.1 \mathrm{M}$ solution of EDTA.

HEDTA (Hydroxyethylene diamine triacetic acid) HEDTA (AR No. 1) made by $\mathrm{KOCH}$-Light laboratories Ltd. was used to prepare $0.1 \mathrm{M}$ solution.

CYDTA (Trans 1, 2, cyclohexane diamine tetra acetic acid)

CYDTA made by Fluka A.G, A.R. grade is used to prepare $0.1 \mathrm{M}$ solution by dissolving the compound by adding drop-by-drop ammonia solution and then diluted.

\section{EGTA (Ethylene glycol bis (2-amino ethylene)} $\mathbf{N}, \mathbf{N}^{1}, \mathbf{N}^{11}, \mathbf{N}^{111}$ tetra acetic acid)

EGTA used is from Fluka. A.G. which is dissolved by adding dilute ammonia drop by drop, just sufficient to dissolve before dilution.

\section{Standardization of solutions of EDTA, HEDTA, CYDTYA and EGTA}

$10 \mathrm{~mL}$ of zinc standard solution is diluted to $50 \mathrm{~mL}$ with distilled water, $2 \mathrm{~mL}$ of ammonia buffer $(\mathrm{pH}=10)$, and a few drops of Eriochrome black $\mathrm{T}$ as indicator. The solution is then titrated against the EDTA. Similarly, HEDTA, CYDTA and EGTA are standardized by titrating against zinc standard solution. 


\section{Secondary ligands}

Maltol (3, hydroxyl-2-methyl 4,pyrone)

$\mathrm{KOCH}$ Light Laboratories $0.1 \mathrm{M}$.

Tiron (Catechol 3,5-disulfuric acid disodium salt) Fluka AG. Buch.

Kojic acid BDH, biochemical grade recrystallized from hot alcohol containing animal charcoal.

$\alpha$-Picolinic acid BDH A.R.

\section{Preparation of Erbium solution}

Erbium (III) oxide $\left(\mathrm{Er}_{2} \mathrm{O}_{3}\right)$ obtained from Indian Rare Earth Ltd of purity 99.905 was dissolved in nitric acid, heated on a water bath, cooled and then diluted. The final free acidity is maintained at $0.1 \mathrm{M}$.

\section{Standardization of Erbium solution}

$5 \mathrm{~mL}$ of rare earth solution is diluted to 25 $\mathrm{mL}$ with distilled water. $\mathrm{pH}$ is adjusted to 5.5 using saturated hexamine solution, two drops of xylenol orange indicator is added and then titrated against standard EDTA till the color changes from red to lemon yellow.

\section{Spectrophotometric measurements}

The $\mathrm{pH}$ of Erbium $\left(\mathrm{Er}^{+3}\right)$ solution $(1 \mathrm{~mL})$ is adjusted to 8.0 by $15 \%$ solution of triethanolamine and then made to $10 \mathrm{ml}$ to give the molarity of $1.0 \mathrm{x}$ $10^{-2} \mathrm{M}$, which is same as primary ligand. The spectra of the solutions taken in the range $350-\mathrm{nm}-450 \mathrm{~nm}$, scan rate $60 \mathrm{~nm} /$ minutes.

For mixed complexes same procedure with secondary ligand solution added to give $4 \times 10^{-3}$ to $3 x$ $10^{-2} \mathrm{M}$. The maximum absorbance values recorded for different molar ratios of secondary ligands to the primary complexes. The absorbance values for Erbium are measured at $379 \mathrm{~nm}$ which is the $\lambda_{\text {max }}$ chosen for maximum sensitivity.

\section{RESULTS AND DISCUSSIONS}

The absorbance increases steadily in an almost linear fashion with the increased in concentration of secondary ligand until the molar ratio of $1: 1$ is reached for [secondary ligand]/[primarycomplex], thereafter it increases but the increased is only normal. This pattern proves that the primary complex fully transformed into ternary or mixed ligand complex at the above molar ratio and the stoichiometry of all the ternary complexes of erbium (Er) thus found to be 1:1: 1 as shown in Figure 1 to 4 .

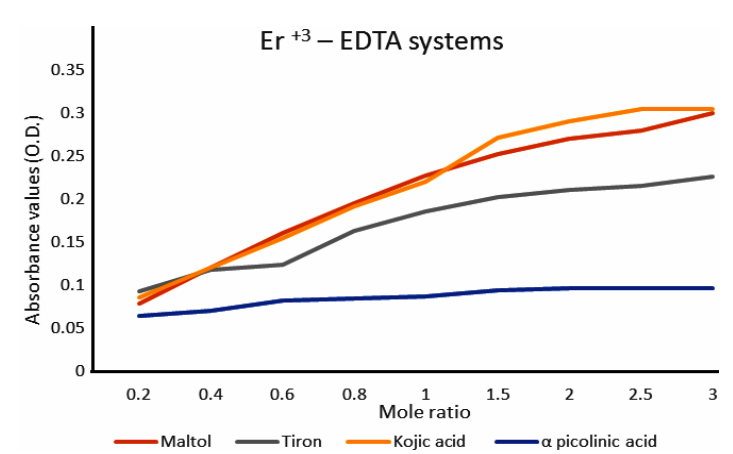

Fig. 1. Absorbance vs. Mole ratio of [Secondary ligand]/ [Erbium-EDTA]

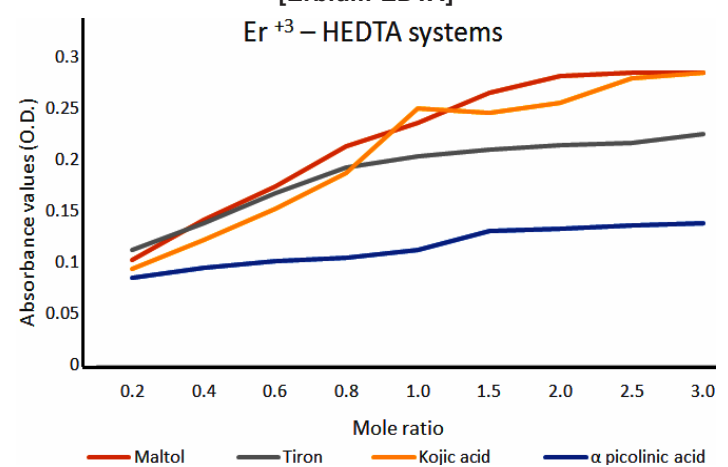

Fig. 2. Absorbance vs. Mole ratio of [Secondary ligand]/ [Erbium-HEDTA]

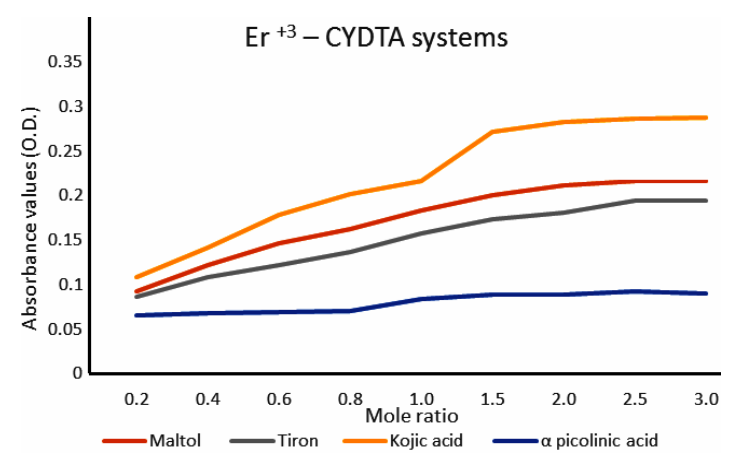

Fig. 3. Absorbance vs. Mole ratio of [Secondary ligand]/ [Erbium-CYDTA]

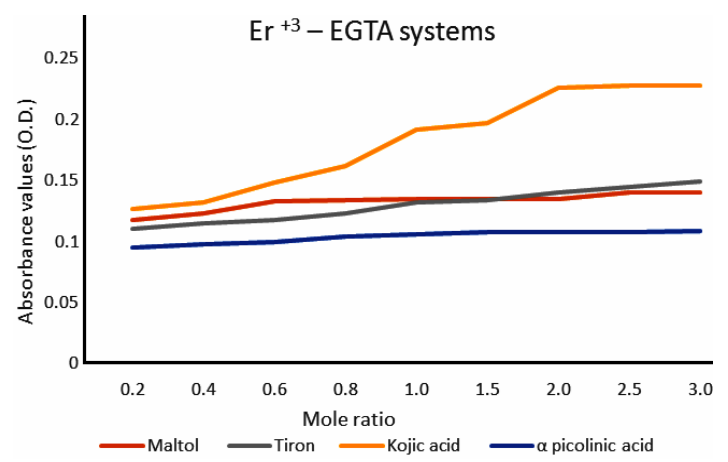

Fig. 4. Absorbance vs. Mole ratio of [Secondary ligand]/ [Erbium-EGTA] 
This result is in line with the Stoichiometry of ternary complexes of Neodymium and Europium using tryptophan and sodium citrate has constant composition of M-L-L' (1:1:1) reported by Wang,,$^{10}$. Mixed ligand systems of Holmim-triethylene tetraamin hexaacetic acid-Maltol shows the stoichiometry of $2: 1: 2 .{ }^{11}$

The absence of interaction between the ligands and $4 \mathrm{f}$ orbitals of metal ions supported by the observation that the complexing groups have only small effects on the position of these sharp well define absorption bands in the UV region as evident from the absorption spectra Figure 5.

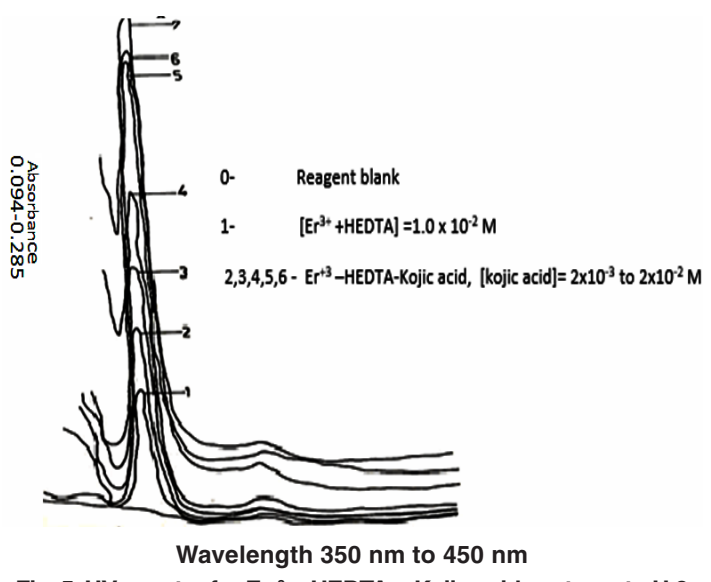

Fig. 5. UV-spectra for $\mathrm{Er}^{+3}+\mathrm{HEDTA}+$ Kojic acid system at $\mathrm{pH} 8$

A study of rare earth complexes as reported in research article ${ }^{4}$ shows that the coordination number for these elements varies from six to nine in aqueous solutions. In present study we found the common coordination number for Erbium is eight as evident from the results which shows 1:1:1 stoichiometry for all the ternary complexes of Erbium studied, where the primary ligands are hexadentate and the secondary ligands are bidentate.

The hyperchromic effect seen when ternary complexes are formed is evident from increase in molar extinction coefficient of ternary complexes (M-Lp-Ls) compare to primary complexes which are calculated as \% increase in the molar extinction coefficient and are given in Table 1. Formation of ternary complexes with large bathochromic shift and increase in molar absorptivity compared to binary complexes is a useful tool in micro determination of rare earths as reported by Belsare, G.W. ${ }^{12}$ The $\%$ increase in molar coefficients of mixed ligand complexes of Erbium are found to be significant which can facilitates the analytical measurements for micro determination of Erbium ions.

The order of hyperchromic effect with respect to secondary ligands in the ternary complexes of Erbium for all the systems studied are Kojic acid $>$ Maltol $>$ Tiron $>\alpha$-Picolinic acid as evident from the Fig. 6 . The ternary complexes of HEDTA and CYDTA shows enhancement of stabilities over those of EDTA/EGTA as evident from the Logk's values calculated and given in Table 2 which can be explained in terms of electrostatic effect. In the case of HEDTA and CYDTA the secondary ligand has to combine with a neutral primary chelate while in EDTA/EGTA, it has to face uninegative metal- EDTA/ EGTA chelate.

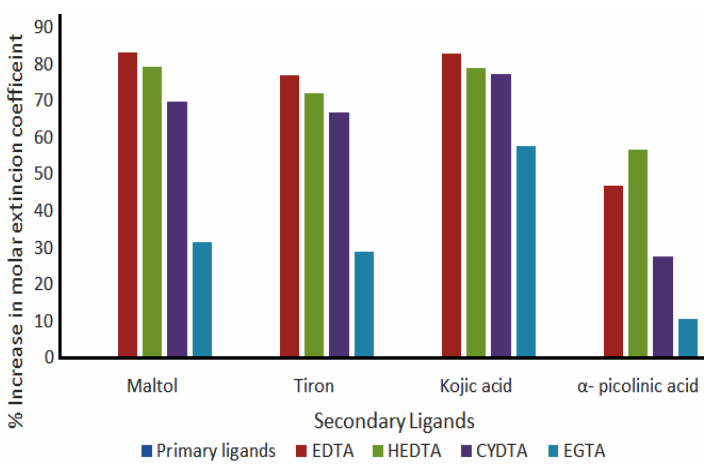

Fig. 6. Percentage increase in molar extinction coefficient of ternary complexes of Erbium

Table 1: \%Increase in molar extinction coefficient (Em) of mixed ligand complexes of Erbium with different primary ligands (LP) and secondary ligands (SL)

\begin{tabular}{lcccc}
\hline Secondary ligands & $\begin{array}{c}\text { \%increase in Em with } \\
\text { EDTA }\left(\mathrm{L} \mathrm{mol} \mathrm{cm}^{-1}\right)\end{array}$ & $\begin{array}{c}\text { \%increase in } \mathrm{Em} \text { with } \\
\text { HEDTA }\left(\mathrm{L} \mathrm{mol} \mathrm{cm}^{-1}\right)\end{array}$ & $\begin{array}{c}\text { \%increase in Em with } \\
\text { CYDTA }\left(\mathrm{L} \mathrm{mol} \mathrm{cm}^{-1}\right)\end{array}$ & $\begin{array}{c}\text { \%increase in Em with } \\
\mathrm{EGTA}\left(\mathrm{L} \mathrm{mol} \mathrm{cm}^{-1} \mathrm{~cm}^{-1}\right)\end{array}$ \\
\hline Maltol & 83.2 & 79.1 & 69.9 & 31.52 \\
Tiron & 76.9 & 72.1 & 66.7 & 29.0 \\
Kojic acid & 82.8 & 79.0 & 77.3 & 57.7 \\
$\alpha$-picolinic acid & 47 & 56.6 & 27.7 & 10.44 \\
\hline
\end{tabular}


Table 2: Comparison of conditional stability constant $\left(\log \mathbf{k} \mathbf{s}^{1}\right)$ with corrected stability constants

\begin{tabular}{|c|c|c|c|c|c|c|c|c|}
\hline Primary ligands & $\begin{array}{l}\log k^{1} \text { for } \\
E^{+3}-E D T A\end{array}$ & Logks $1 / p k$ & $\begin{array}{l}\text { Log ks }^{1} \text { for } \\
\text { Er }^{+3} \text {-HEDTA }\end{array}$ & Logks $1 / p k$ & $\begin{array}{l}\log _{k s}{ }^{1} \text { for } \\
\mathrm{Er}^{+3}-\mathrm{CYDTA}\end{array}$ & $\operatorname{Logks}^{1} / \mathrm{pk}$ & $\begin{array}{l}\operatorname{Log~ks}^{1} \text { for } \\
\mathrm{Er}^{+3}-\mathrm{EGTA}\end{array}$ & $\operatorname{Logks}^{1 / p k}$ \\
\hline Maltol & 2.55 & 0.29 & 3.37 & 0.38 & 3.27 & 0.37 & 3.02 & 0.35 \\
\hline Tiron & 3.22 & 0.42 & 3.48 & 0.45 & 2.61 & 0.34 & 3.34 & 0.38 \\
\hline Kojic acid & 2.72 & 0.35 & 2.84 & 0.36 & 3.48 & 0.44 & 3.16 & 0.40 \\
\hline$\alpha$-picolinic acid & 2.86 & 0.57 & 3.06 & 0.60 & 2.52 & 0.5 & 2.89 & 0.57 \\
\hline
\end{tabular}

In establishing the order of stability for ligands where ligand has one basic functionality, the ratio of $\log k_{s}{ }_{s}^{1} / \log k H=A$, where $\log k H$ is the measure of basicity of ligand, thus $A$ will reflect the affinity of ligand anion more faithfully. ' $A$ ' can be named as the affinity parameter and the stability order for the ligands can be established on the basis of $A$ values and not on their $\operatorname{Logk}_{s}{ }^{1}$ values. The conditional stability constants corrected using pKs values of secondary ligands as by Shetty, S. ${ }^{8}$. The $\mathrm{K}_{\mathrm{s}}{ }^{1}$ and corrected stability constant values are plotted against the secondary ligands as shown in Fig. 7. Comparison of these corrected stability constants shows that for Erbium-EDTA-Maltol, Er-HEDTA-Maltol, Er-CYDTA-Maltol and ErbiumEGTA-Maltol systems the values of $A$, ratio of $\operatorname{LogK}_{\mathrm{s}} 1 / \mathrm{pKs}$ remains almost constant at 0.32 $(+0.04$ to -0.04$)$.

$$
\text { —EDTA —HEDTA } \because \text { CYDTA —EGTA }
$$

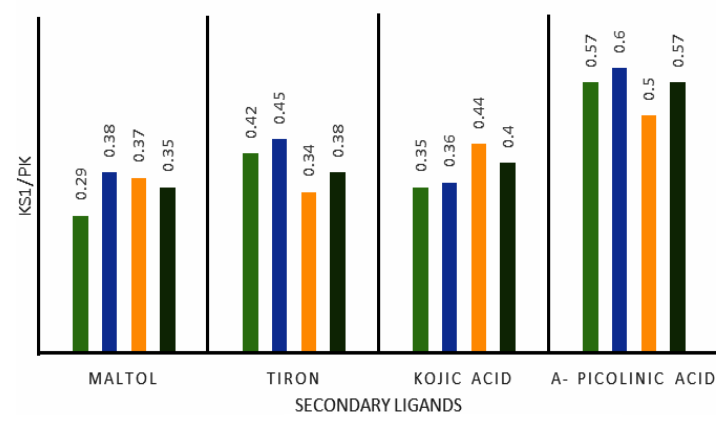

Fig. 7. Log $k_{s}^{1 / p ~ K ~ f o r ~ m i x e d ~ l i g a n d ~ c o m p l e x e s ~ o f ~ E r b i u m ~}$

Thus, using the above parameter, the order of stability with the series of ligands in different systems is deduced as EDTA and HEDTA:
$\alpha$-Picolinic acid $>$ Tiron $>$ Kojic acid $>$ Maltol and CYDTA and EGTA: $\alpha$-Picolinic acid > Kojic acid > Tiron > Maltol.

\section{CONCLUSION}

The stability order clearly brings out the preference of the Erbium ion for nitrogen donor ligands over oxygen donor ligand. This is in direct contrast to the Holmium-EDTA with bidentate ligands where the preference is to oxygen donor ligands as per the findings of Shetty S.Y.

The complexing agents used shows significant hyperchromic effect which can be exploited for the determination of Erbium ion at very low concentration.

It is possible to separate, estimate and analyze the Erbium ions $\left(\mathrm{Er}^{+3}\right)$ using these versatile complexing agents.

The study will continue to establish relation between stability order for different rare earth elements with same primary and secondary ligands and ionic radii of metal ions.

\section{ACKNOWLEDGMENT}

I thank Dr. R. M. Sathe, Dr M. Sunderesan of Analytical Chemistry Division, Bhabha Atomic Research Center Trombay, Mumbai 400085.

\section{Conflicts of Interest}

The author declare no conflict of interest.

\section{REFERENCES}

1. Taketatsu, T.; Yoshida, S.; J. Inog. Nucl. Chem., 1973, 35, 881-890.

2. Cam, T.; Irez, G.; Aydin, R. J. Chem. Eng. Data., 2011, 56(5), 1813-1820.

3. Shetty, S.Y.; Sathe, R. M. J. Tala., 1976, 23, 46-47.

4. Khan, M.M. T.; Reddy, P. R. J. Inorg. Nucl. Chem., 1972, 34, 967-972.

5. Vekhande, C.; Munshi, K.N. Ind. J. Chem., 1976, 14, 189-191.

6. Zade,B.A.; Kalbende, P. P.; Umerkar,S.M.; Belsare, W.G. J. Chem., 2012, 9(4), 2394-2406.

7. Dhepe, S.A.; Zade, B.A. J. Chem., 2011, 8
(3), 1264-1274.

8. Shetty, S.Y.; Sathe, R.M.; Ind. J. Chem., 1983. 22A, 452-453.

9. Vogel, A.I.; A Text. B. Quant. Inorg. Analy., 1989, 5, 328-352.

10. Wang, X.-C,; Gao, Q. K.; Wang, K. J.; Wang. R.; Chem. Papers., 2012, 66, 188-193.

11. Sathe, R. M.; Shetty, S.Y.; Ind. J. of Chem., 1986, 25A, 494-495.

12. Belsare, G.W.;Zade, A.B.; Kalbende, P.P.; Belsare, P.U.; Der Phar. Chemi., 2012, 4 1226-1238. 